\title{
The correlation between C-reactive protein and toxic granulation of neutrophils in the peripheral blood
}

\author{
A van de Vyver, E F Delport, M Esterhuizen, R Pool
}

Background. During inflammation, the serum concentrations of granulocyte colony-stimulating factor (G-CSF), plasma interleukin-6 (IL-6), and C-reactive protein (CRP) increase. A positive correlation between CRP and the percentages of neutrophils exhibiting toxic granulation during inflammation has been demonstrated, and that the fluctuations of CRP and toxic granulation of neutrophils were similar.

Objectives. We studied whether grading of toxic granulated neutrophils can be used as a surrogate marker for infection or inflammation, and also be an easier method than previously described methods.

Materials and methods. We graded 357 consecutive peripheral blood slides from patients on whom a full blood count with differential count and CRP level was performed, according to intensity of toxic granulation in the neutrophil population, according to a newly proposed grading system.
Results. The CRP range was between 1 and $530.3 \mathrm{mg} / \mathrm{l}$. The results confirm the association between a rise in CRP and progressive intensity of toxic granulation in neutrophils in peripheral blood. Kruskal-Wallis equality of populations rank test showed a statistically significant difference between the graded categories $(p=0.0001)$. The Trend test was also statistically significant $(p=0.000)$.

Conclusion. The proposed system can be applied to patients with inflammatory or infectious conditions, where grading of toxic granulation of neutrophils can possibly be used as a surrogate marker to assess infection or inflammation and their response to treatment. It may be of particular use in cases where traditional infectious or inflammatory markers cannot be used, owing to inherent problems associated with the respective conditions.

S Afr Med J 2010; 100: 442-444.
Severe inflammatory and infectious conditions can elicit different reactions including pyogenic, granulomatous, lymphocytic and also non-inflammatory changes. The most common tissue response in acute inflammation is dominated by neutrophils. ${ }^{1}$ During inflammatory or infectious conditions, mature neutrophils can display toxic granulation (TG). TG is characterised as dark blue-black granules in the cytoplasm of mature neutrophils and is especially helpful in predicting acute bacterial infection. ${ }^{1,2}$ The appearance of TG is the result of an increase in acid mucosubstance in azurophilic granules, which stains more prominently than under normal circumstances. ${ }^{3-5}$

These toxic granules are formed in the promyelocyte stage during neutrophil maturation, and contain antimicrobial substances. ${ }^{6}$ The acidic mucosubstance accumulated in the toxic granules also has the potential to acidify phagosomes. These toxic granules enhance bactericidal activity, since bacteria in phagosomes are killed more effectively at a $\mathrm{pH}$ of 5.5 than at a $\mathrm{pH}$ of $7 .^{3}$ The formation of toxic granulated neutrophils (TGN) is induced by granulocyte colony-stimulating factor (G-CSF), which increases the number of granulocyte precursors in bone marrow that activate mature granulocytes. ${ }^{7}$ During inflammation, the serum concentrations of G-CSF and plasma interleukin-6 (IL-6) increase. ${ }^{8,9}$

Department of Haematology, University of Pretoria, and NHLS

A van de Vyver, BSc, MB ChB, PG (Dip) TM

M Esterhuizen, MB ChB, MMed (Haemat)

R Pool, MB ChB, MMed (Haemat)

Department of Internal Medicine, University of Pretoria

E F Delport, MB ChB, MMed (Intern Med)

Corresponding author: A van de Vyver (annievdvyver@gmail.com)
The serum levels of C-reactive protein (CRP) increase during inflammation, infection and tissue damage, in response to stimulation by cytokines, mainly interleukin-6., ${ }^{2,70-14} \mathrm{CRP}$ is the most commonly used parameter to evaluate inflammation or infection, ${ }^{15}$ but is not diagnostic on its own, and must be interpreted with the clinical picture and other investigations. ${ }^{10}$

The use of neutrophil left-shift parameters as indicators for infective disease has been evaluated previously, but has limited value. Toxic changes such as vacuolisation and the presence of Dohle bodies occur in infectious diseases and have been associated with high levels of CRP. ${ }^{16}$ However, morphological changes in neutrophils, such as TG, have a high sensitivity (80\%) for predicting infection. ${ }^{16}$ A combination of more than one neutrophil left-shift parameter will increase the sensitivity. ${ }^{3}$ The relationship between infectious diseases and TG has been recognised for many years; however, the efficacy of grading the intensity of TG in predicting infectious or inflammatory conditions is controversial. ${ }^{2}$ A previous study demonstrated a positive correlation between CRP and the percentage of neutrophils exhibiting TG during inflammation. The researchers counted 200 mature neutrophils and expressed them as a percentage of toxic granulated neutrophils in mature neutrophil group. ${ }^{2} \mathrm{CRP}$ and TG of neutrophils had similar fluctuations. ${ }^{17}$ These findings suggest that evaluation of TG of neutrophils could be as useful in monitoring inflammation as CRP. Kabutomuri et al. showed lower CRP levels with a high percentage of toxic granulated neutrophils; whereas patients with normal liver function showed good correlation. ${ }^{2}$ They suggested that the percentage of toxic granulated neutrophils is more useful than CRP in diagnosing inflammation in patients with abnormal liver function. ${ }^{2}$

In our study, we designed a new TG grading scale to assess whether grading of toxic granulated neutrophils can be used as 
a surrogate marker for infection or inflammation. This method is less labour-intensive and less time-consuming than the methods used in previous studies.

\section{Materials and methodology}

Ethical approval was obtained from the Faculty of Health Science Research Ethics Committee, University of Pretoria, on 29 April 2009, protocol number 61/2009.

From patients seen in Steve Biko Academic Hospital, Pretoria, we examined 357 consecutive peripheral blood slides, where the treating clinician had requested a full blood count, with a differential white cell count, as well as CRP. Neonates and patients with suspected or confirmed acute leukaemia were excluded. Peripheral blood smears were made from venous blood samples collected in vacutainer tubes, containing ethylene diamine tetra-acetic acid (EDTA), and stained with Wright Giemsa stain. Morphological examination of neutrophils was performed at magnifications of 500x and $1000 x$.

We evaluated a possible correlation between the serum level of CRP and the graded intensity of observed TG in neutrophils, according to number and stain intensity. A minimum of 50 cells were evaluated on every slide, and an average impression was obtained and categorised. Neutrophils with TG were defined by the presence of dark blue to purple cytoplasmic granules in the neutrophils (Fig. 1).

The TG intensity was graded into 5 categories $(0,1+, 2+$, $3+$ and $4+$ ), as set out in Table I. Serum levels of CRP were measured by a nephelometric method utilising CRP antibodies (Beckman Coulter DX1). The CRP distribution between the 5 TG categories was evaluated to assess a possible trend or relationship.

Statistical analysis was performed using STATA 9 statistical package (STATA Corporation). The results were expressed in box and whisker plots analysis to determine the mean, minimum and maximum CRP levels, in each graded category. Statistical difference between the categories was evaluated using Kruskal-Wallis equality of populations rank test. A trend test was also performed.

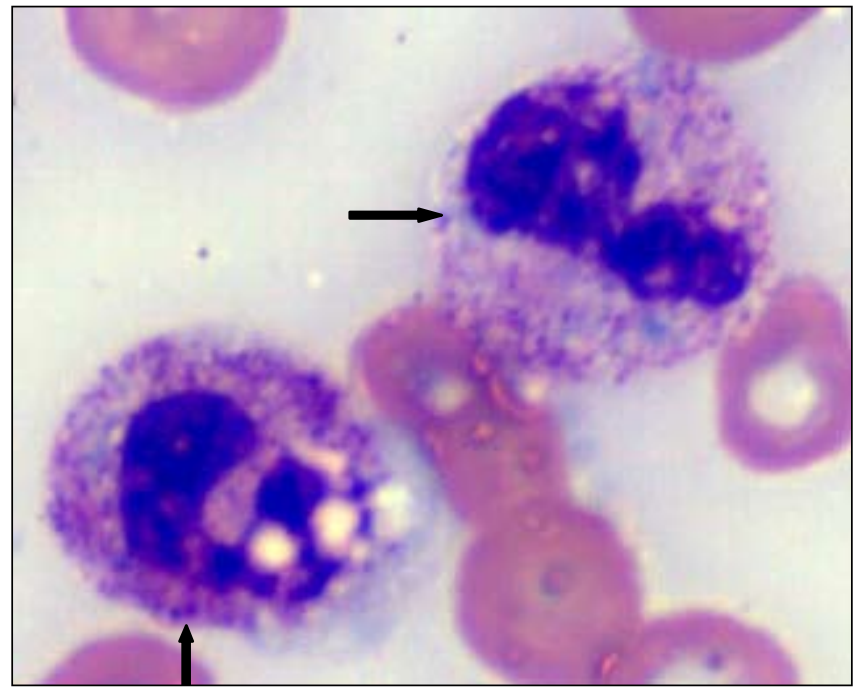

Fig. 1. Toxic granulation of neutrophils in the peripheral blood.

\section{Table I. CRP characteristics in TG categories}

$\begin{array}{ll}0 & \text { Normal granulated neutrophils } \\ 1 & \begin{array}{l}\text { Scattered granules in the cytoplasm with an increase } \\ \text { in the stain intensity }\end{array} \\ 2 & \begin{array}{l}\text { Increased numbers of granules in the cytoplasm with } \\ \text { an increase in stain intensity }\end{array} \\ 3 & \text { Numerous granules present in the cytoplasm with } \\ & \text { intense blue black staining properties }\end{array}$

After the initial analysis into 5 categories of graded toxic granulation $(0,1+, 2+, 3+$ and $4+)$, the categories were combined and all specimens were grouped as 0 (no TG observed), 1 (1+ and 2+ TG) and 2 (3+ and 4+ TG), to improve statistical significance.

\section{Results}

In total, 357 consecutive peripheral smears were evaluated. The CRP figures ranged between 1 and $530.3 \mathrm{mg} / \mathrm{l}$. A statistically significant difference between the graded categories was demonstrated $(p<0.001)$, as well as the trend between incremental TG categories $(p<0.001)$ (Fig. 2, Table II). These results confirm a trend in the rise of CRP in proportion to the TG of neutrophils in peripheral blood. This could be applied to patients with inflammatory or infectious conditions, where the grading of TG of neutrophils can possibly be used as a marker of the severity of infection or inflammation.

\section{Discussion}

The relationship between TG in mature neutrophils and high CRP levels in infectious disease and inflammation has been

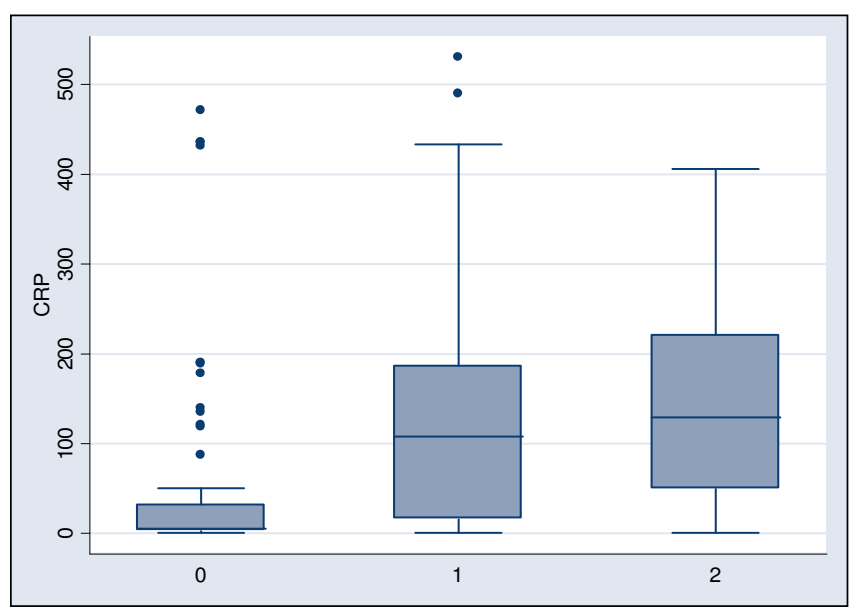

Fig. 2. Data depicted as box and whisker plots.

\section{Table II. CRP characteristics in TG categories}

\begin{tabular}{llll}
\hline Group & 1 & 2 & 3 \\
\hline$N$ & 61 & 225 & 71 \\
CRP mean (mg/l) & 5.3 & 108.2 & 129.3 \\
Minimum CRP & 1 & 1 & 1 \\
Maximum CRP & 470.8 & 530.3 & 405.8 \\
\hline
\end{tabular}


described. ${ }^{1,3,6}$ However, the relationship between the intensity of TG and CRP levels has not been recognised. In our present study, the graded intensity of TG in neutrophils was evaluated using a newly designed scale which was then compared with the CRP values in patients with possible inflammatory or infectious conditions.

Controversy exists regarding the use of white blood cell quantitative parameters, and qualitative changes as indicators of acute bacterial infection. ${ }^{1,3,17}$ Determining the intensity of TG is labour-intensive and the reproducibility is potentially poor, owing to inter- and intra-observer variability. Grading TG is dependent on technique, training and the experience of the technologist or haematologist. It has been suggested that the total white blood cell count and absolute neutrophil count should be monitored because these parameters yield more reproducible results. Despite these difficulties, most laboratories still use morphological changes in neutrophils (i.e. TG and vacuolisation) on peripheral blood smears as a diagnostic tool, especially to predict acute pyogenic infections. ${ }^{18-20}$ Liu et al. described the usefulness of these morphological changes, finding that the presence of TG was more predictive of bacteraemia than the total leukocyte count. ${ }^{17}$ Sabah et al. found toxic changes in neutrophils to be highly sensitive, which was confirmed by Al-Gwaiz and Babay, ${ }^{1}$ who found a sensitivity of $78 \%$ for detecting acute bacterial infection. Kabutomori et al. found that movements of both CRP and TG of neutrophils were similar when evaluating blood results in 8 patients with infective conditions, and suggested that measuring TG of neutrophils is useful in monitoring inflammatory processes. No mention was made of any correlation with CRP values. ${ }^{3}$

Grading the intensity in comparison with determining the percentage of toxic granulated neutrophils is potentially a less labour-intensive strategy for determining the presence of infection and inflammation. Grading TG may be particularly useful in patients for whom traditional infectious or inflammatory markers cannot be used.

Accepted inflammation markers can be of limited use in certain patient populations. CRP levels can be markedly elevated with tissue necrosis that is not necessarily associated with infection. Conversely, normal to moderate elevation can be seen in patients with infection and underlying liver disease and certain auto-immune diseases such as systemic lupus erythromatosus, scleroderma and ulcerative colitis. ${ }^{21}$ The use of total white cell count as a marker can also be biased by corticosteroid use, which produces falsely elevated counts, as it prevents migration to the tissue pool. ${ }^{22}$ The erythrocyte sedimentation rate (ESR), long used as a broad screen for inflammatory processes, can be falsely normal or low in the presence of iron deficiency. Newer markers such as procalcitonin (PCT) can also be confounded by the presence of renal failure, which will produce false high levels. Therefore, a tool is needed that can be used to monitor infection in conditions affecting liver and renal function, associated with underlying inflammatory processes, with possible concurrent corticosteroid therapy. These conditions include auto-immune diseases and HIV-1 infection. The degree of TG can be a useful adjunct in this setting, where most other markers have been confounded.

We found a statistically significant trend between grading the intensity of toxic granulation in neutrophils and a rise in CRP. Statistically significant differences in CRP were found between the graded TG categories. Morphological changes in neutrophils, especially TG, are therefore helpful in evaluating disease severity in patients with underlying inflammatory or infectious conditions.

\section{Conclusion}

The above results confirm a trend in the rise of CRP, as the intensity of TG of neutrophils in peripheral blood increases. This finding could be applied in patients with inflammatory or infectious conditions, where grading of TG of neutrophils can possibly be used as a marker to assess infection or inflammation severity. This method could be especially valuable in cases where the infective markers do not reflect the clinical picture.

\section{References}

1. Al-Gwaiz LA, Babay HH. The diagnostic value of absolute neutrophil count, band count and morphologic changes of neutrophils in predicting bacterial infections. Med Princ Pract 2007; 16: 344-347

2. Kabutomori $\mathrm{O}$, Kanakura $Y$, Watani $Y$. Toxic granulation neutrophils and $\mathrm{C}$-reactive protein Arch Intern Med 2001; 160: 3326-3327.

3. Kabutomori O, Kanakura Y, Watani Y. Induction of toxic granulation in neutrophils by granulocyte colony-stimulating factor. Eur J Haematol 2002; 69: 187-188.

4. McCall CE, Caves J. Disfunction of human neutrophils during severe infection. Clin Res 1970; 18: 49 .

5. McCall CE, Katayama I, Cotran RS, et al. Lysosomal and ultrastuctural changes in human "toxic" neutrophils durin bacterial infection. I Exp Med 1969; 129: 267-282.

6. Kim YS, Park HH, Rhee HW, et al. Neutrophils with toxic granulation shows high fluorescence with Bis (ZN2+-dipicolylamine) complex. Ann Clin Lab Sci 2009; 39(2): 114-119.

7. Kabutomori O, Kanakura $Y$, Watani $Y$. Movement of toxic granulation neutrophils and C-reactive protein in inflammatory processes. Am J Med 2002; 112: 595-596.

8. Kawakami M, Tsutsumi H, Kumakawa T, et al. Levels of serum granulocyte colonystimulatin factor in patients with infections. Blood 1990, 76: 1962-1964.

9. Struzyna J, Pojda Z, Braun B, Chomicka, Sobiczewska E, Wremel J. Serum cytokine levels (IL-4, IL-6, IL-8, G-CSF) in burned patients. Burns 2000; 21(6): 437-440.

10. Pepys MB, Hirschfield. C-reactive protein: a critical update. J Clin Invest 2003; 111: 1805-1812.

11. Heney D, Lewis IJ, Evans SW, Banks R, Bailey CC, Whicher JT. Interleukin-6 and its relationship to C-reactive protein and fever in children with febrile neutropenia. J Infect Dis 1992; 165: 886-890.

12. Katz JA, Mustafa MM, Bash RO, Cash JV, Buchanan GR. Value of C-reactive protein determination in the initial diagnostic evaluation of the febrile, neutropenic child with cancer Pediatr Infect Dis J 1992; 11: 708-712.

13. Engel A, Mack E, Kern P, Kern WV. An analysis of interleukin-8, interleukin-6 and C-reactive protein serum concentrations to predict fever, Gram-negative bacteraemia and complicated infection in neutropenic cancer patients. Infection 1998; 26: 213-221.

14. Lehrnbecher T, Venzon D, de Haas M, Chanock SJ, Kuehl J. Assessment of measuring circulating levels of interleukin-6, interleukin-8, C-reactive protein, soluble Fc gamma circulating levels of interleukin-6, interleukin-8, C-reactive protein, soluble Fc gamm
receptor type III, and mannose-binding protein in febrile children with cancer and neutropenia. Clin Infect Dis 1999; 29: 414-419.

15. Fischer CL, Gill C, Forrester MG, et al. Quantitation of "acutephase proteins" postoperatively. Value in detection and monitoring of complications. Am J Clin Pathol 1976; 66: 840e6.

16. Seebach JD, Morant R, Ruegg R, Seifer LB, Fehr J. The diagnostic value of the neutrophil left shift in predicting inflammatory and infectious diseases. Am J Clin Pathol 1997; 107: 582-591.

17. Liu CH, Lehan C, Speer ME. Early detection of bacteremia in an outpatient clinic. Pediatrics 1985; 75: 827-831.

18. Mathy K, Koeptke JA. The clinical usefulness of segmented vs slab neutrophil criteria for differential leukocyte count. Am J Clin Pathol 1979; 6: 947-958.

19. Benez EI. Hematologic response to acute inflammation: the band neutrophil revisited. Tex Med 1990; 86: 26-28.

20. Hoyer ID. Leucocyte differential. Mayo Clin Proc 1993; 86: 26-28.

21. Pepys MB, Hirschfield M. C-reactive protein: a critical update. J Clin Invest 2003; 111: 18051812.

22. Hoffbrand AV, Catovsky D, Tuddenham EGD. Postgraduate Haematology. 5th ed. Oxford, UK Blackwell Publishing, 2005: 286.

Accepted 17 February 2010. 